\title{
Thermal shock experiment of beryllium exposed to intense high energy proton beam pulses
}

\author{
K. Ammigan, ${ }^{1}$ S. Bidhar, ${ }^{1}$ P. Hurh, ${ }^{1}$ R. Zwaska, ${ }^{1}$ M. Butcher, ${ }^{2}$ M. Calviani, ${ }^{2}$ M. Guinchard,${ }^{2}$ \\ R. Losito, ${ }^{2}$ V. Kuksenko, ${ }^{3}$ S. Roberts, ${ }^{3}$ A. Atherton, ${ }^{4}$ G. Burton, ${ }^{4}$ O. Caretta, ${ }^{4}$ T. Davenne, ${ }^{4}$ \\ C. Densham, ${ }^{4}$ M. Fitton, ${ }^{4}$ P. Loveridge,${ }^{4}$ and J. O'Dell ${ }^{4}$ \\ ${ }^{1}$ Fermi National Accelerator Laboratory, Batavia, Illinois, USA \\ ${ }^{2}$ CERN, Geneva, Switzerland \\ ${ }^{3}$ University of Oxford, Oxford, United Kingdom \\ ${ }^{4}$ Rutherford Appleton Laboratory, Didcot, United Kingdom
}

(Received 8 November 2018; published 4 April 2019)

\begin{abstract}
Beryllium is a material extensively used in various particle accelerator beam lines and target facilities, as beam windows and, to a lesser extent, as secondary particle production targets. With increasing beam intensities of future multimegawatt accelerator facilities, these components will have to withstand even greater thermal and mechanical loads during operation. As a result, it is critical to understand the beaminduced thermal shock limit of beryllium to help reliably operate these components without having to compromise particle production efficiency by limiting beam parameters. As part of the RaDIATE (radiation damage in accelerator target environments) Collaboration, an exploratory experiment to probe and investigate the thermomechanical response of several candidate beryllium grades was carried out at CERN's HiRadMat facility, a user facility capable of delivering very-high-intensity proton beams to test accelerator components. Multiple arrays of thin beryllium disks of varying thicknesses and grades, as well as thicker cylinders, were exposed to increasing beam intensities to help identify any thermal shock failure threshold. Real-time experimental measurements and postirradiation examination studies provided data to compare the response of the various beryllium grades, as well as benchmark a recently developed beryllium Johnson-Cook strength model.
\end{abstract}

DOI: 10.1103/PhysRevAccelBeams.22.044501

\section{INTRODUCTION}

Beryllium is currently widely used as the material of choice for critical accelerator components such as beam windows and secondary particle production targets in various accelerator beam lines and target facilities. One of the main challenges facing beam windows and targets exposed to high energy high-intensity proton beams is the induced thermal shock in the material from beam pulses of short duration [1]. Dynamic stress waves are generated due to the high-temperature gradient and differential expansion set up by the nearly instantaneous temperature jump in the localized region of the beam spot [2]. These dynamic propagating stress waves, driven by inertia and superimposed on the already present quasistatic stresses in the material, can be large enough to push the material beyond its yield point to cause plastic deformation or crack

Published by the American Physical Society under the terms of the Creative Commons Attribution 4.0 International license. Further distribution of this work must maintain attribution to the author(s) and the published article's title, journal citation, and DOI. initiation and even failure if the crack propagates through the material. Therefore, it is essential to thoroughly understand the material's thermal shock response and identify any failure limits in order to successfully design and reliably operate critical beam-intercepting accelerator components such as beam windows and targets.

With the increasing beam intensities of future multimegawatt accelerator facilities, beam-intercepting components are expected to operate in even more extreme environments, potentially pushing materials close to their thermal and structural limits. The Long Baseline Neutrino Facility at Fermilab [3] is an example of such a facility, where intense proton beams (up to $2.4 \mathrm{MW}, 120 \mathrm{GeV}, 1.5 \times 10^{14}$ protons per pulse, beam $\sigma_{\text {rms }} \sim 1.5 \mathrm{~mm}, 9.6 \mu$ s pulse length) will interact with beam windows and targets to produce intense neutrino beams for the Deep Underground Neutrino Experiment (DUNE). The induced stresses from the desired beam parameters currently exceed a very conservative target design stress limit based on static beryllium yield stress at a low temperature and strain rate [4]. Hence, to avoid compromising particle production efficiency by limiting beam parameters, it is important to experimentally identify the thermal shock limits and failure mechanisms of the material at high strain rates and temperatures. 
The thermal shock response of beryllium has previously been studied within the fusion energy community, where it is the plasma facing material of choice for the International Thermonuclear Experimental Reactor fusion test reactor [5]. Linke et al. [6] and Spilker et al. [7] have used electron beams to mimic the high energy density deposition and induced thermal shock expected on the inner walls of a fusion tokamak. Microstructural studies were then performed to evaluate material degradation and resistance to thermal shock from varying loading cycles. The induced thermal shock in these studies, however, occurred only in a very thin layer below the surface of the beryllium, analogous to the expected operating conditions in fusion reactors.

On the other hand, for high energy proton beams $(>100 \mathrm{MeV})$ in accelerator target facilities, thermal shock is typically induced through the volume of the beamintercepting material. The resulting thermal and dynamic stress fields generated are consequently different from the surface thermal shock case in previous fusion reactor studies. Therefore, it is essential to use high energy proton beams to replicate the operating conditions of target facility components by simultaneously imposing a high strain rate and high-temperature conditions in a localized volume of the beam-intercepting material.

A beryllium in-beam experiment (HRMT-24) at CERN's HiRadMat facility was therefore proposed and carried out within the RaDIATE Collaboration [8] framework to impose strong thermal shock effects from high-intensity proton beams. The HiRadMat facility [9] is a user facility at CERN which can deliver a high-intensity pulsed beam to an experimental area where accelerator materials and devices can be tested under a controlled environment. The facility uses the $440 \mathrm{GeV} / c$ beam, extracted from the Super Proton Synchrotron (SPS), with adjustable beam parameters (bunch intensity, number of bunches, and bunch spacing) to meet the needs of each experiment. With the HiRadMat beam parameters, it was possible to expose beryllium to thermal shock levels not previously encountered in existing accelerator facilities while also pushing the material to its limit.

The main objectives of the experiment were to expose and compare various commercially available grades of beryllium to high-intensity proton beams in order to (i) identify and quantify thermal shock limits, (ii) explore the threshold of failure modes (crack initiation or fracture) under controlled localized strain rates and temperatures, and (iii) benchmark advanced highly nonlinear numerical simulations by collecting real-time and postirradiation experimental material response data.

\section{BEAM-INDUCED THERMOMECHANICAL RESPONSE IN BERYLLIUM}

The HiRadMat facility has the capability to deliver proton beams of up to $4.9 \times 10^{13}$ protons per $7.2 \mu$ s pulse (maximum of 288 bunches with $1.7 \times 10^{11}$ protons per bunch) with an energy of $440 \mathrm{GeV}$ and a Gaussian beam spot size ranging from 0.1 to $2 \mathrm{~mm}$ beam sigma [9]. The number of bunches ( $0.375 \mathrm{~ns}$ bunch length) in each pulse, the bunch spacing $(25,50,75$, or $150 \mathrm{~ns})$, and the beam spot size can be controlled before the beam is extracted to the experimental area to induce the desired thermal shock effect in the experiment. For beryllium, the beam parameters were carefully chosen to push the material to its solid limit (close to the melting temperature) with a single $7.2 \mu \mathrm{s}$ beam pulse (288 bunches with 25 ns bunch spacing). Several MARS Monte Carlo [10] particle-matter simulations were performed to determine the required beam parameters to achieve the desired conditions. MARS volumetric heat deposition results were then input into ANSYS ${ }^{\circledR}$ and LS-DYNA ${ }^{\circledR}$ finite element analysis (FEA) software to evaluate the expected temperature rise and resulting mechanical response in the material.

For a thin beryllium disk, interacting with a single highintensity HiRadMat beam pulse of $0.3 \mathrm{~mm}$ beam sigma and $4.9 \times 10^{13}$ protons per $7.2 \mu \mathrm{s}$ pulse, the FEA results indicate a peak temperature of $1050^{\circ} \mathrm{C}$, close to beryllium's melting temperature $\left(1285^{\circ} \mathrm{C}\right)$. With the steep Gaussian radial temperature gradient that is induced in the material over a very short timescale, large dynamic stresses are expected to be generated in the disk because of thermal shock. For the current LBNF design beam parameters, the temperature jump in beryllium is expected to be around $200^{\circ} \mathrm{C}$, which pushes the material beyond its elastic limit during a single pulse. Operating a beryllium beam window in the elastic-plastic regime is somewhat unexplored and uncertain, and, therefore, the HiRadMat beam parameters in this experiment were chosen accordingly to probe the failure threshold and limit, stretching from the elastic to plastic deformation regime and up to close to the material's melting point. The primary goal is to identify the real experimental limit of the material and, hence, avoid compromising beam parameters, to maximize the physics benefits.

During the design of the experiment, limited and extrapolated temperature- and strain-rate-dependent beryllium material properties from the literature [11] were input into the structural FEA analyses to evaluate the beam-induced stresses and strains. The LS-DYNA ${ }^{\circledR}$ elasticviscoplastic material model (MAT_106) [12] was implemented, and Fig. 1 shows 2D axisymmetric contour plots of effective strains and stresses for a 0.75 -mm-thick beryllium disk at the end of the beam pulse and upon cooldown back to room temperature.

Large effective strains of up to $3.6 \%$ are induced at the end of the beam pulse [Fig. 1(a)], and, after the disk cools down back to room temperature, a peak residual effective strain of up to $3 \%$ remains [Fig. 1(c)]. The residual strain is exhibited by permanent out-of-plane deformations (on the order of a few micrometers) in the beam spot region on both 


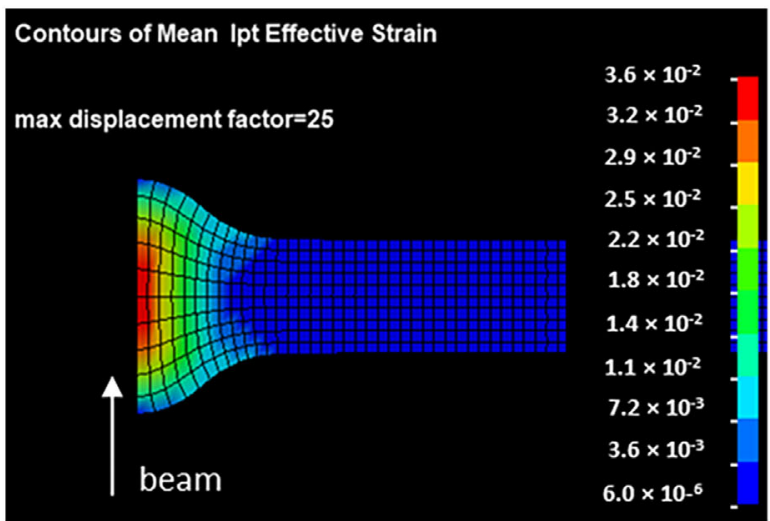

(a)

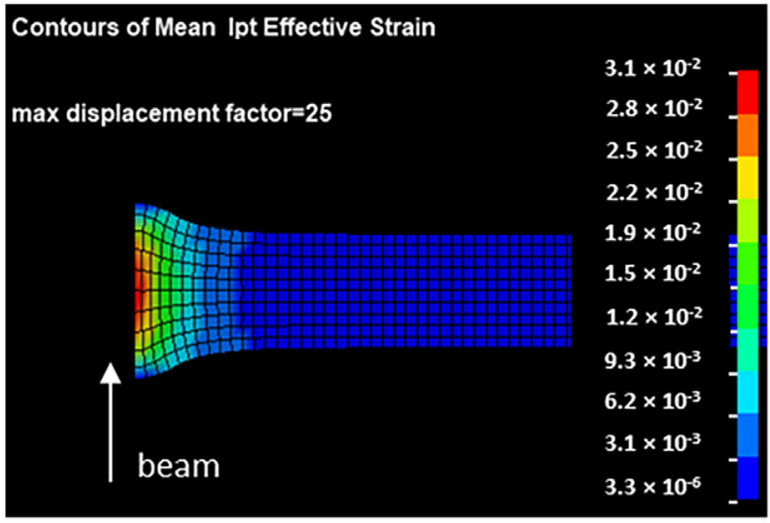

(c)

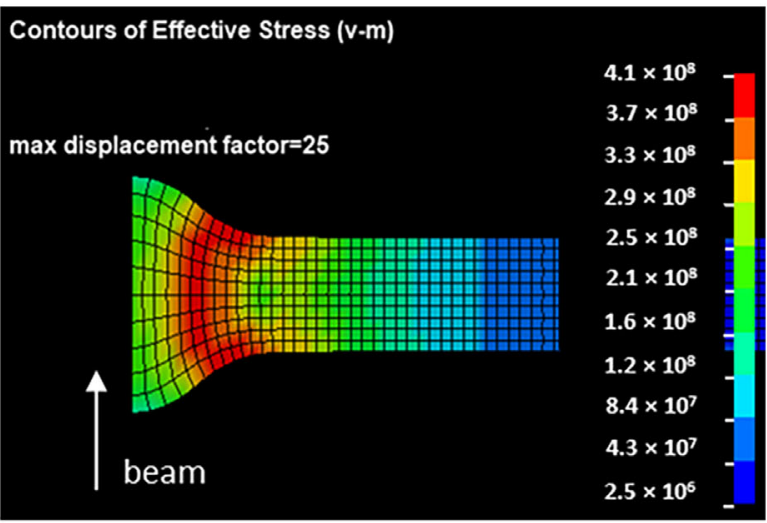

(b)

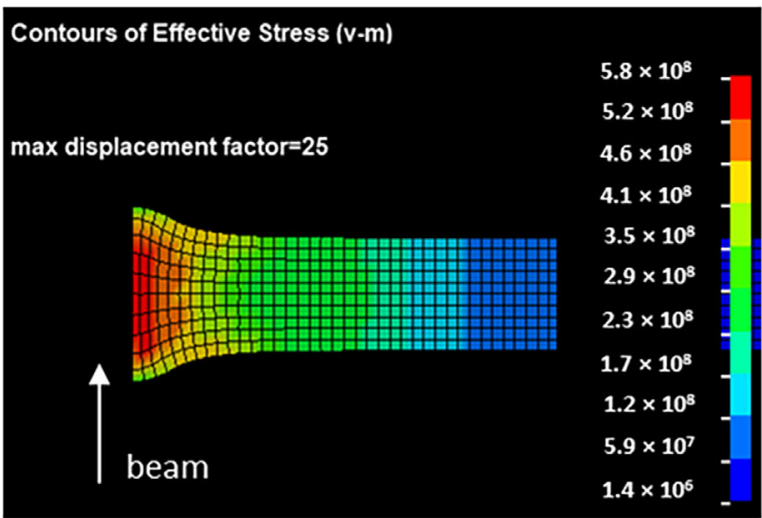

(d)

FIG. 1. Beam-induced effective strain and stress in 0.75-mm-thick Be disks: (a) strain and (b) stress (Pa) at the end of the beam pulse $\left(7.2 \mu \mathrm{s}, \Delta T \sim 1050^{\circ} \mathrm{C}\right)$ and (c) strain and (d) stress (Pa) at room temperature after cooldown $\left(0.3 \mathrm{~s}, T \sim 25^{\circ} \mathrm{C}\right)$.

faces of the disk. This highly localized plastic deformation is caused when the instantaneously heated region is constrained from expanding by the surrounding cooler material, during the short beam pulse (much shorter than the heat dissipation time). This sets up the thermal shock effect, and dynamic stress waves start to propagate in both the axial and radial directions of the disk. The residual 3\% effective strain for the case simulated in Fig. 1 exceeds the reported failure strain ( $2 \%$ for S-200-F at RT) in the literature [13]. This suggests that, for this set of beam parameters where the temperature of the beryllium is rapidly brought close to its melting point, internal cracking or perhaps fracture of the disk near the beam spot region can be expected. Also note that the stresses upon cooldown

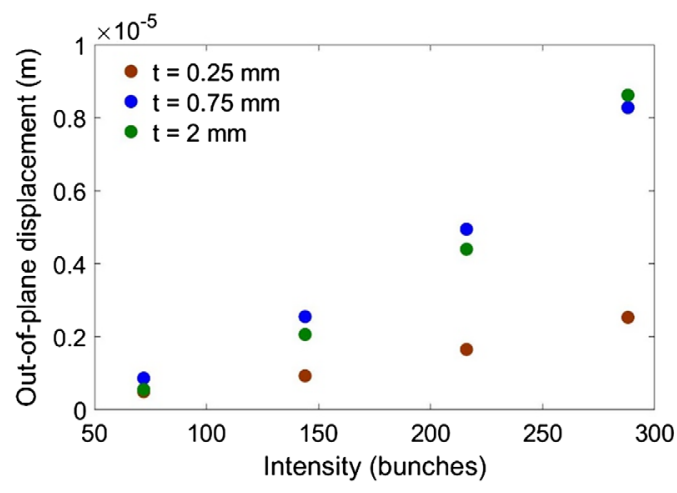

(a)

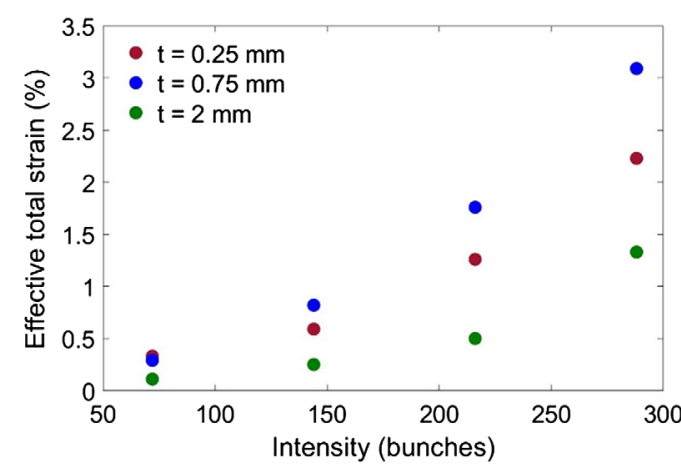

(b)

FIG. 2. Simulation results showing (a) permanent out-of-plane deformation and (b) residual effective strain of a beryllium disk as a function of the beam intensity $(\sigma=0.3 \mathrm{~mm})$ after cooldown back to room temperature following a single beam pulse. 


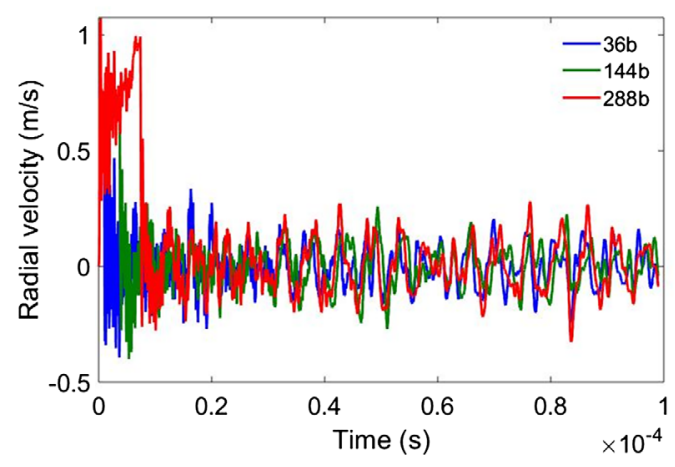

(a)

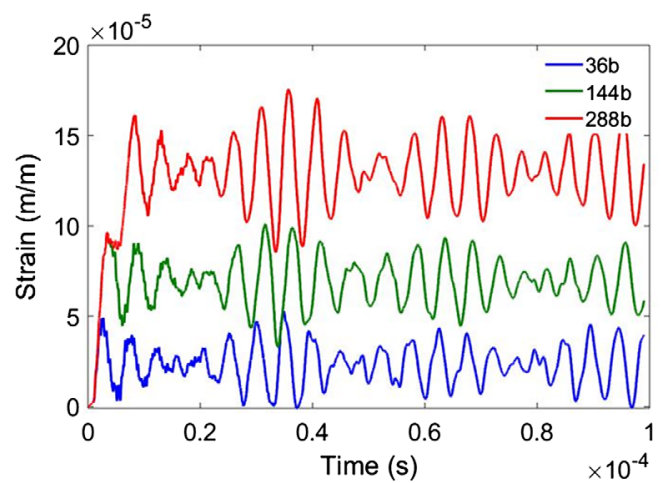

(b)

FIG. 3. Beam-induced dynamic effects from the HiRadMat beam $(\sigma=0.3 \mathrm{~mm})$ as a function of the beam intensity $\left(1.7 \times 10^{11}\right.$ protons per bunch), (a) radial velocity and (b) axial strain.

exceed the material's reported ultimate tensile stress of about $365 \mathrm{MPa}$ [13].

Figure 2 shows out-of-plane deformation and effective total strain simulation results as a function of beam intensities and beryllium disk thicknesses, after being subjected to a single beam pulse and allowed to cool back down to room temperature.

It is shown that, at even lower beam intensities ( 72 bunches with $1.2 \times 10^{13}$ protons in $1.8 \mu \mathrm{s}$ ) where the peak temperature jump is about $330^{\circ} \mathrm{C}$, some residual permanent out-ofplane deformation $(\sim 0.5 \mu \mathrm{m})$ is expected. Therefore, by subjecting several arrays of beryllium specimens of varying thicknesses to increasing beam intensities, possible thermal shock failure thresholds or limits can be identified.

Dynamic stresses, driven by inertial effects during the short beam pulse and superimposed on the quasistatic stresses, further increase the peak stresses in the material. Figure 3 shows simulation results of dynamic effects on the circumferential surface of a thick beryllium cylinder $(\mathrm{r}=20 \mathrm{~mm}, \mathrm{~L}=30 \mathrm{~mm})$ upon interaction with the HiRadMat beam incident $2 \mathrm{~mm}$ from its cylindrical edge.

\section{HRMT-24 EXPERIMENTAL SETUP}

The experimental chamber consisted of four vertically separated arrays of specimens with each array exposed to single or multiple beam pulses of varying intensities. Figure 4 shows the overall experimental setup with the experimental chamber installed on the HiRadMat mobile table. The chamber sat on a vertical lift tower which was remote controlled and dc-motor actuated and with a positioning precision of $\pm 100 \mu \mathrm{m}$ to allow for accurate

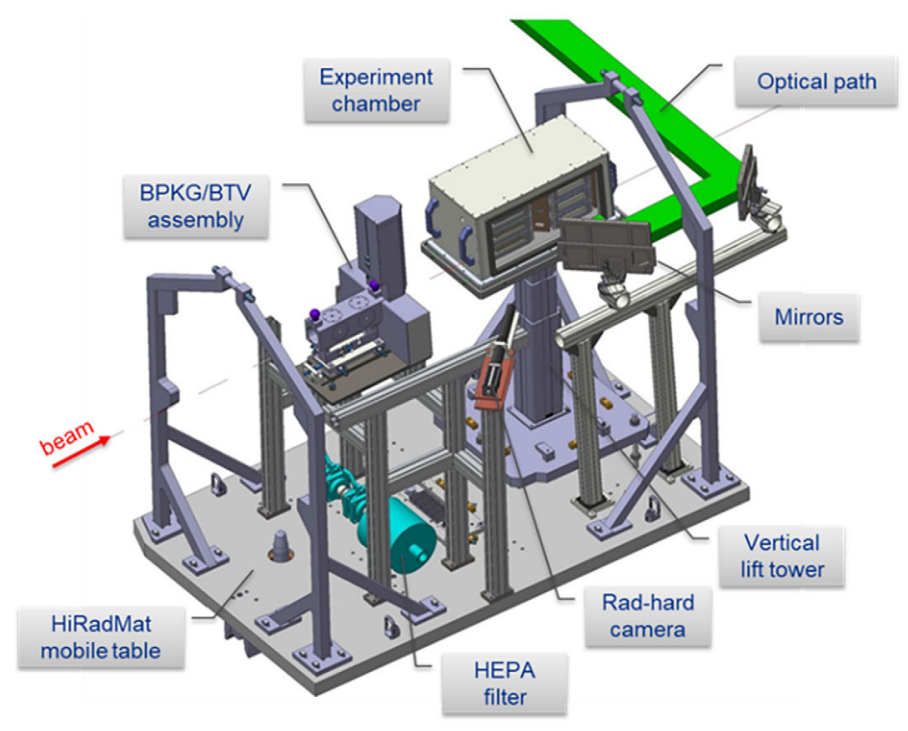

(a)

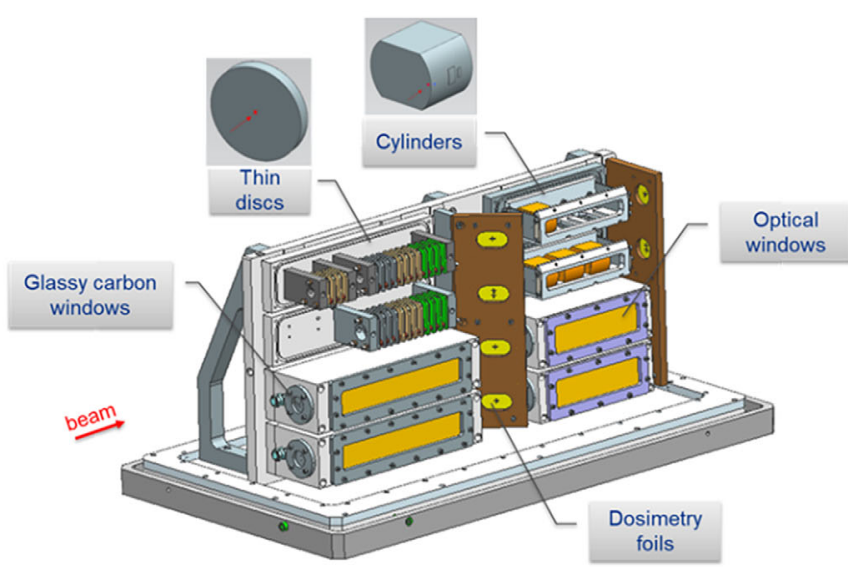

(b)

FIG. 4. Experimental setup. (a) Outer chamber installed on the HiRadMat mobile table, and (b) interior of the outer chamber showing specimens and inner containment boxes. 


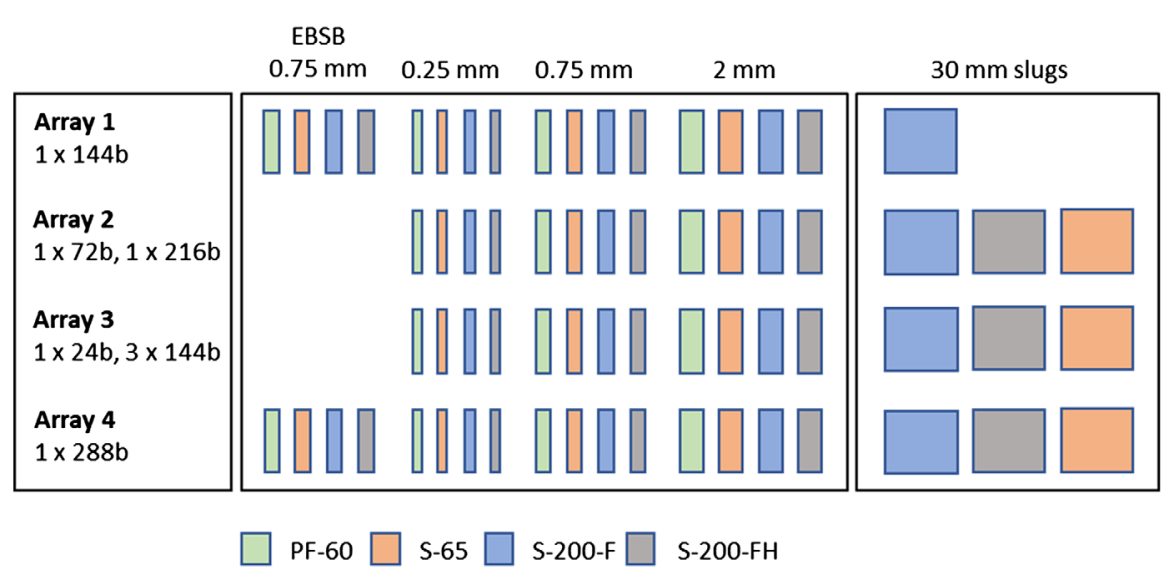

FIG. 5. Test matrix showing specimen type, size, grade, and beam intensity.

vertical alignment of each specimen array to the incident beam. Small apertures on the upstream and downstream ends of the chamber allow the proton beam to enter and exit.

Because of the toxicity of beryllium and the potential for radioactive contamination upon beam interaction, the experimental chamber was based on a double (dynamic) containment design to ensure proper containment of the beryllium. An outer containment chamber enclosed several hermetically sealed inner containment boxes that contained the various specimens. This allowed for the internal air volume of the outer chamber to be continuously evacuated by an air pump via a HEPA filter (tube connections not shown in Fig. 4) during the experiment. This maintained a lower pressure within the outer chamber and ensured that no airborne particulate escaped from the outer containment chamber during the experiment. The HEPA filter, analyzed upon the completion and disassembly of the experiment, provided a check on containment breach of the hermetically sealed inner boxes.

A beam position and profile monitor assembly [14,15], positioned and aligned upstream of the experimental chamber, provided beam diagnostics by measuring the location and profile of each beam pulse. As a secondary beam diagnostic tool, dosimetry films precisely positioned inside of the experimental chamber and in conjunction with a radiation-hard camera mounted on the mobile table monitored beam alignment in real time as the films were exposed by the beam. Optical windows allowed visual access for a radiation-hard camera and a high-resolution camera, as well as for a laser Doppler vibrometer (LDV) system used to measure the surface displacement and vibration of specific specimens. Mirrors mounted accurately on the mobile table provided the optical path to the high-resolution camera, LDV, and data acquisition systems, positioned behind shielding blocks in an adjacent tunnel (TT61) to the HiRadMat experimental area (TNC tunnel).

Thin disk specimens ranging from 0.25 to $2 \mathrm{~mm}$ thick were enclosed in the upstream boxes in each array, while the downstream boxes contained instrumented thicker specimens (30-mm-thick slugs) for real-time measurements. The inner boxes were hermetically sealed with optical windows and glassy carbon beam windows. The dosimetry films were oriented by $45^{\circ}$ to the beam axis to allow for imaging with the radiation-hard camera. A second set of dosimetry films positioned at the downstream end of the box, perpendicular to the beam, were also analyzed at the end of the experiment to provide further beam position information.

The design specimen test matrix, as illustrated in Fig. 5, consisted of four commercially available grades of beryllium (S-200-F, S-200-FH, S-65, and PF-60 [16]) with differing impurity content, consolidation processes, and mechanical strength properties. The strength properties of the beryllium grades are given in Table I (PF-60 grade strength data not available in the literature). A few thin disks were precharacterized by electron backscatter diffraction (EBSD) analysis and were selectively placed in arrays 1 and 4 . The thin disk specimens, with a $200 \mathrm{~nm} \mathrm{rms}$ surface finish, were analyzed during postirradiation examination (PIE) work after the completion of the experiment.

Real-time thermal and mechanical response measurements were obtained from the slugs that were enclosed in the downstream inner boxes of each array. The slugs were aligned so that the beam impacted the front face of the slug

TABLE I. Material data for various grades of beryllium (quasistatic test conditions) [17].

\begin{tabular}{lccc}
\hline \hline Grade & $\begin{array}{c}\text { Yield strength } \\
(\mathrm{MPa})\end{array}$ & $\begin{array}{c}\text { Tensile strength } \\
(\mathrm{MPa})\end{array}$ & $\begin{array}{c}\text { Elongation } \\
(\%)\end{array}$ \\
\hline S-200-FH & 327 & 455 & 4.3 \\
S-65 (transverse) & 293 & 412 & 8.7 \\
S-65 (longitudinal) & 290 & 391 & 5.7 \\
S-200-F & 244 & 368 & 6.3 \\
$\quad$ (transverse) & & 341 & 3 \\
S-200-F & 249 & & \\
$\quad$ (longitudinal) & & & \\
\hline \hline
\end{tabular}




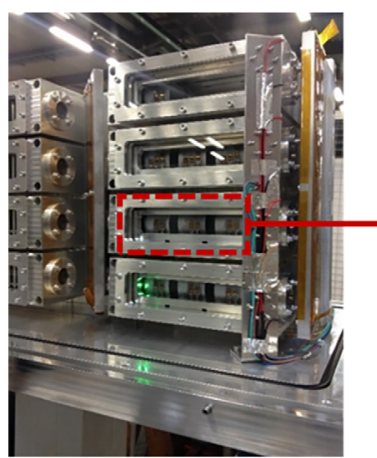

(a)

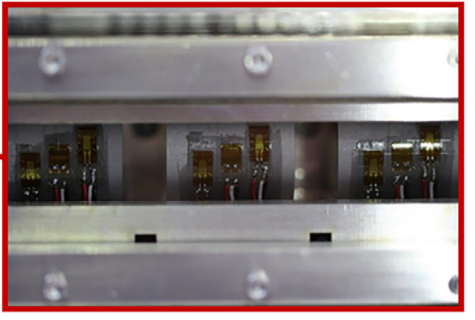

(b)

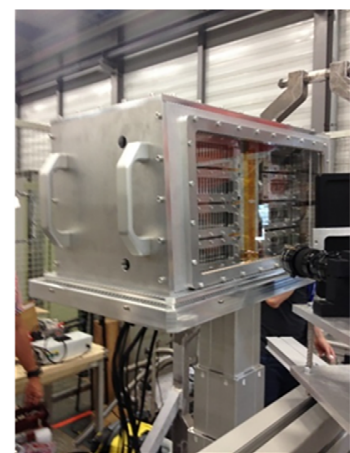

(c)

FIG. 6. Installation and testing of the HRMT-24 setup. (a) Inner containment boxes of slug specimens, (b) strain and temperature gages on beryllium slugs, and (c) outer containment chamber.

$3.2 \mathrm{~mm}$ away from the cylindrical edge in order to obtain larger signals during the online measurements. Strain and temperature gages, attached to the beryllium slugs, measured the circumferential strain and surface temperature immediately upon beam interaction. An LDV laser, directed perpendicular to the slug's cylindrical surface, measured the radial vibration and deformation in real time. The S-200-F grade was arbitrarily chosen for the LDV measurement, as the expected thermomechanical dynamic response differences between the different beryllium grades was somewhat unknown prior to the experiment. Note that the PF-60 grade was omitted as a slug due to its unavailability in thicknesses greater than $3 \mathrm{~mm}$. Figure 5 also provides the design beam intensities for each array. Array 2 would receive two beam pulses separated vertically on the specimens, and array 3 would receive multiple beam pulses at the same location on the specimens to explore plastic deformation accumulation due to cyclic loading.

Figure 6 shows the slug inner boxes and the experimental chamber assembly. Prior to installation in the tunnel, the components of the experimental chamber, instrumentation, and data acquisition systems were all assembled and tested on the mobile table in the HiRadMat service building (BA-7). Using fiducials and laser tracking systems, an alignment and a survey of the experimental chamber were performed on a dummy experimental table in BA-7 to accurately position the chamber with respect to the theoretical beam line position. Figures 6(a) and 6(b) show the strain and temperature gages attached to the cylindrical surface of the slugs enclosed in their inner containment boxes and mounted on the vertical base plate. A mockup of the optical path was also created in BA-7 to test the highresolution camera and the LDV signal strength from the specimen surface [green LDV laser on the upstream slug in array 4 visible in Fig. 6(a)]. Figure 6(c) shows the outer containment chamber assembled to the vertical lift tower, as well as the radiation-hard camera mounted and oriented perpendicular to the angled dosimetry films.

\section{EXPERIMENTAL PARAMETERS AND RESULTS}

Table II summarizes the extracted beam parameters for each pulse imposed on the four arrays. A total of 11 beam pulses were sent to the experiment, with the bunch intensity averaging about $1.3 \times 10^{11}$ protons per bunch. The beam spot shape was slightly elliptical with an average beam sigma of $\sigma_{x}=0.3 \mathrm{~mm}$ and $\sigma_{y}=0.25 \mathrm{~mm}$.

Because of accelerator operational constraints at the time of our experiment, lower beam intensities than the design specifications $\left(\sigma_{x, y}=0.25 \mathrm{~mm}, 1.7 \times 10^{11}\right.$ protons per bunch) were delivered to the specimen arrays. As a result, instead of the desired $1000^{\circ} \mathrm{C}$ maximum beam-induced temperature jump in array 4 , only a $640^{\circ} \mathrm{C}$ jump was achieved due to the larger beam sigma and lower average bunch intensity of the beam extracted to the experiment. The $640{ }^{\circ} \mathrm{C}$ temperature jump over the $5.4 \mu$ s beam pulse attained in this experiment was, however, still larger than what beryllium has been previously exposed to during operation in current accelerator facilities.

TABLE II. Extracted beam pulses to the experiment.

\begin{tabular}{llcccc}
\hline \hline \multirow{2}{*}{$\begin{array}{l}\text { Pulse } \\
\text { no. }\end{array}$} & Array & \multicolumn{2}{c}{ Bunches } & \multicolumn{2}{c}{ Protons on Beam sigma } \\
per pulse & target & $\sigma_{x}(\mathrm{~mm})$ & $\sigma_{y}(\mathrm{~mm})$ \\
\hline 1 & 3 & 24 & $3.20 \times 10^{12}$ & 0.30 & 0.20 \\
2 & 2.1 & 36 & $4.72 \times 10^{12}$ & 0.27 & 0.21 \\
3 & 2.2 & 72 & $9.51 \times 10^{12}$ & 0.31 & 0.23 \\
4 & 1 & 144 & $1.87 \times 10^{13}$ & 0.28 & 0.26 \\
5 & 3 & 144 & $1.85 \times 10^{13}$ & 0.30 & 0.31 \\
6 & 3 & 144 & $1.82 \times 10^{13}$ & 0.31 & 0.24 \\
7 & 3 & 144 & $1.86 \times 10^{13}$ & 0.30 & 0.29 \\
8 & 3 & 144 & $1.75 \times 10^{13}$ & 0.30 & 0.27 \\
9 & 3 & 144 & $1.93 \times 10^{13}$ & 0.30 & 0.27 \\
10 & 3 & 144 & $1.93 \times 10^{13}$ & 0.30 & 0.27 \\
11 & 4 & 216 & $2.79 \times 10^{13}$ & 0.30 & 0.27 \\
\hline \hline
\end{tabular}




\section{A. Online thermomechanical measurements}

The 30-mm-thick beryllium slugs at the downstream end of each array [Fig. 6(b)] were included in the experiment to provide real-time measurements of the strain, temperature, and radial vibration or displacement upon interaction with the beam. The quasistatic strain and temperature evolution immediately after the beam pulse was recorded with strain and temperature gages ( $4 \mathrm{kHz}$ sampling frequency), while the dynamic radial vibrational response $(4 \mathrm{MHz}$ sampling frequency) of the slugs was acquired with the LDV. Because of the availability of only one LDV system, only the response of the upstream-most slug (S-200-F) in each array was measured.

Figure 7 shows the temperature response, measured by temperature sensors (HBM TT-3/100), on the cylindrical surface of the slugs located in array 3 (pulse 5, 144 bunches) and array 4 (pulse 11, 216 bunches). Shortly after the beam pulse, the temperature on the surface rises to a maximum and drops back down to room temperature within one second. As expected, a higher peak temperature was recorded for the slugs located in the highest beam intensity array [Fig. 7(b)-array 4]. However, a distinctive temperature response for each of the beryllium grades in each array was observed, with the S-65 grade consistently showing higher temperatures, followed by the S-200-FH and S-200-F grades. This may intrinsically be explained by potential differences in the thermal conductivity of the different beryllium grades, but a closer look at the data also suggests that higher energy deposition and thus higher peak temperatures were likely induced in the downstream slugs due to the particle shower generated from the upstream slugs. This can be inferred by the initial peak electrical noise signal (electromagnetic interference), measured shortly after the beam impact. The magnitude of the peak noise signal, shown at time $\sim 0$ in Fig. 7(a), is larger for the most downstream slug S-65, followed by S-200-FH and S-200-F grade slugs, suggestive of particle shower generation from the upstream slugs to the downstream slugs based on the slug ordering in each array. Another plausible explanation is that the slug inner boxes, relative to the experimental box, were slightly misaligned to the beam, leading to the beam impacting the slugs at different distances from the cylindrical edge where the temperature sensors were attached. One can reasonably argue that the beam was closest to the edge of the S-65 slug (downstream end) and furthest away from the S-200-F slug (upstream end).

The circumferential strains induced by the beam were measured using HBM LY11-3/120 linear strain gages attached to the cylindrical surface of the slugs with M-Bond 610 adhesive. Because of the limitation on the sampling frequency $(4 \mathrm{kHz})$ of the available data acquisition system, only the quasistatic strain response was measured instead of the megahertz-range frequency sampling frequency required to capture the dynamic strains. The thermally induced strain measurements were temperature compensated offline after data acquisition and completion of the experiment. Figure 8 shows the strain response of the beryllium slugs from beam pulses 1 and 11, imposed on array 3 and array 4, respectively.

Clearly observed in Fig. 8 is the high strain response right after the beam pulse (few microseconds) followed by decreasing strain as the slug cools back down to room temperature. With the 24 bunches in pulse 1, the induced strain stays within the elastic limit of the material as predicted, and the strain returns to zero after the slug cools down to room temperature. On the other hand, for the 216-bunch case, significant residual strain remains upon cooldown, indicating that the initial induced strain from the beam pushed the material past its yield strength. Because of the varying yield strengths of the different beryllium grades and potentially larger induced temperatures from particle shower generation, the variation observed in the strain response between the grades is expected. However, with the possibility of beam misalignment discussed earlier, it is difficult to extract meaningful comparisons between the grades until exact beam location data are obtained.

The LDV data collected to obtain radial velocity and displacement data from the slugs were, unfortunately, too noisy, as the reflected laser signal from the surface of the beryllium was weaker than expected. This was mainly due

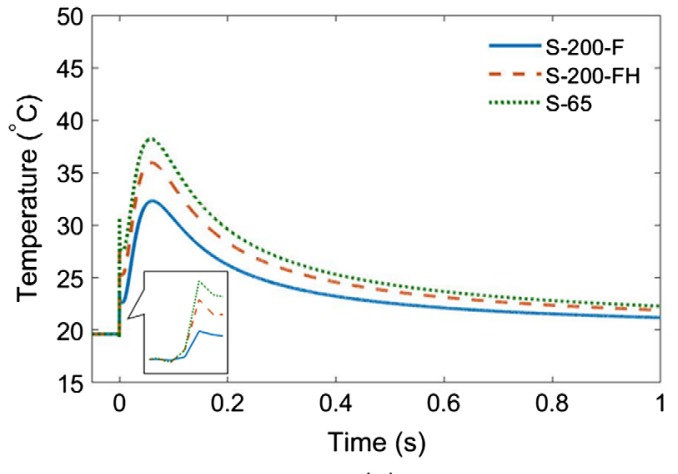

(a)

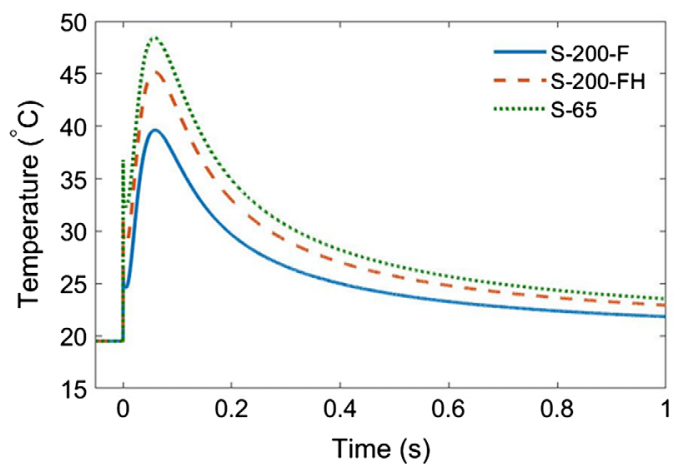

(b)

FIG. 7. Temperature response on a cylindrical surface of beryllium slugs in (a) array 3 (144 bunches) and (b) array 4 (216 bunches). 


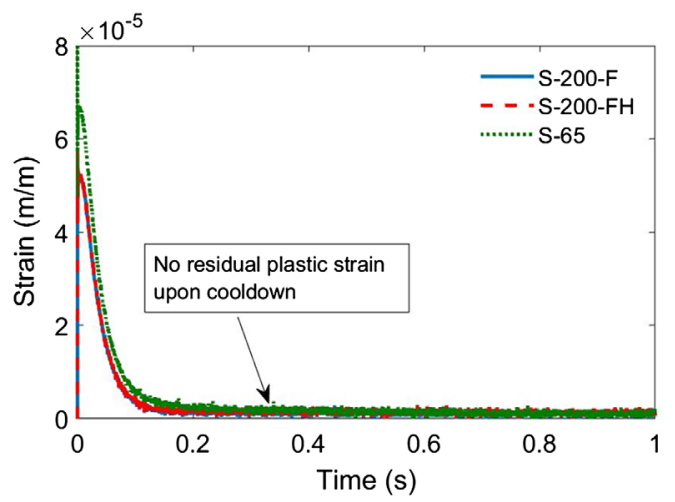

(a)

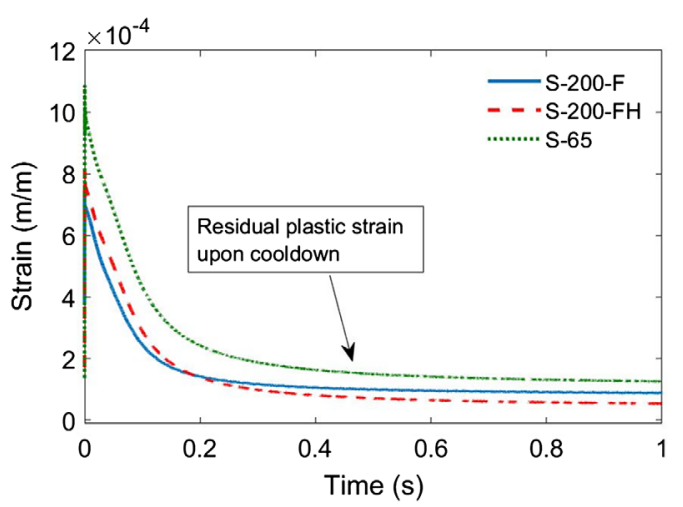

(b)

FIG. 8. Circumferential strain response of beryllium slugs in (a) array 3, 24 bunches, and (b) array 4, 216 bunches.

to the laser having to go through multiple optical windows and mirrors before reaching the LDV sensor located behind shielding blocks in the adjacent tunnel. As a result, the radial vibration and displacement data are not presented in this paper.

\section{B. PIE of thin disk specimens}

After a sufficient cooldown time, the experimental chamber was disassembled at CERN and the thin disk inner containment boxes were retrieved and shipped to the University of Oxford's Department of Materials for PIE work. Light microscopy was first used to inspect the surface of the disk near the beam spot region, and analyses revealed no cracks or fracture. Profilometry was then carried out using an Alicona InfiniteFocus [18] system to measure the out-of-plane deformation [as predicted in Fig. 2(a)] induced in the beryllium disks as a function of thicknesses, beam intensities, and beryllium grades. Figure 9 shows surface deformation profile maps obtained with the profilometer for 0.75 -mm-thick S-65 disks from array 1 (144 bunches) and array 4 (216 bunches). The 216-bunch case [Fig. 9(b)] clearly shows a larger deformation area and peak than the lower-intensity 144-bunch case [Fig. 9(a)].

Figure 10 shows the measured peak out-of-plane deformations of the 0.75- and 2-mm-thick disks from arrays 1, 3, and 4 exposed to different beam intensities. Results for the $0.25 \mathrm{~mm}$ disks are omitted because of the large uncertainty in the surface profile measurement for these disks, mostly due to the surface roughness interfering with the lower measured out-of-plane deformations for these thinner disks. For the same reasons, measurements for disks in array 2, which were exposed to lower pulse intensities, are not included.

As inferred from the plots, the S-200-FH beryllium grade generally shows the least amount of permanent out-ofplane deformation, while the S-200-F grade shows a larger deformation. This can be attributed to the larger yield

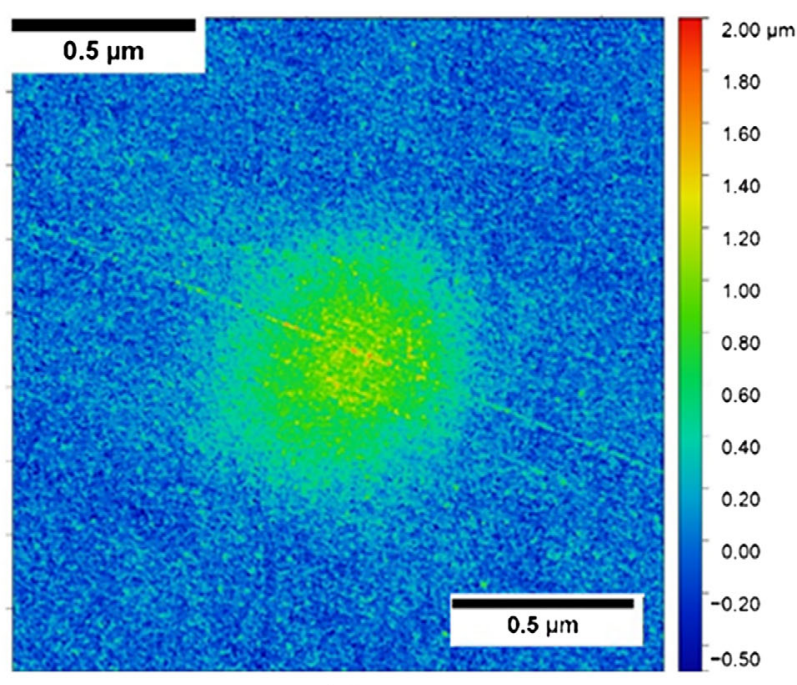

(a)

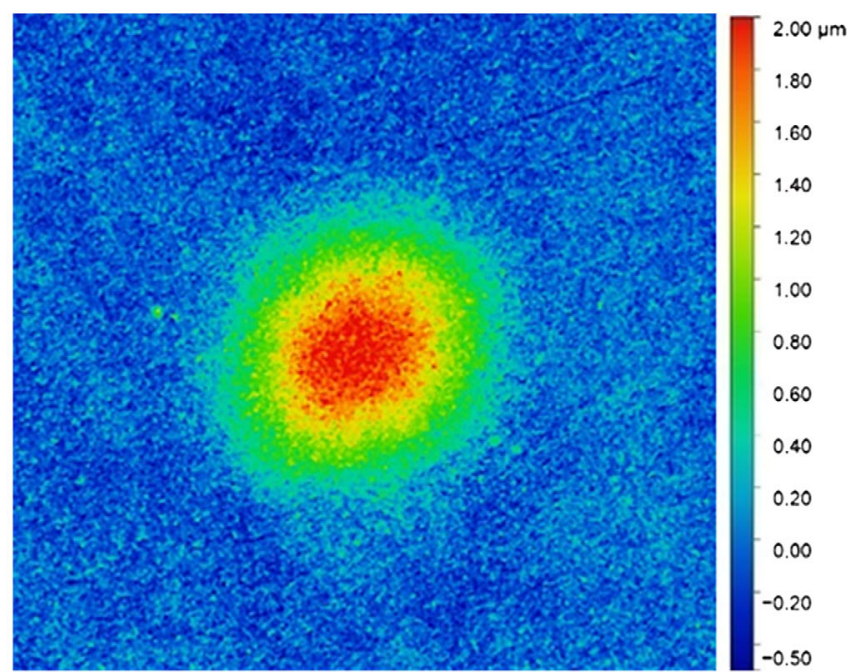

(b)

FIG. 9. Profilometry maps of 0.75-mm-thick S-65 beryllium disks in (a) array 1 (144 bunches) and (b) array 4 (216 bunches). 


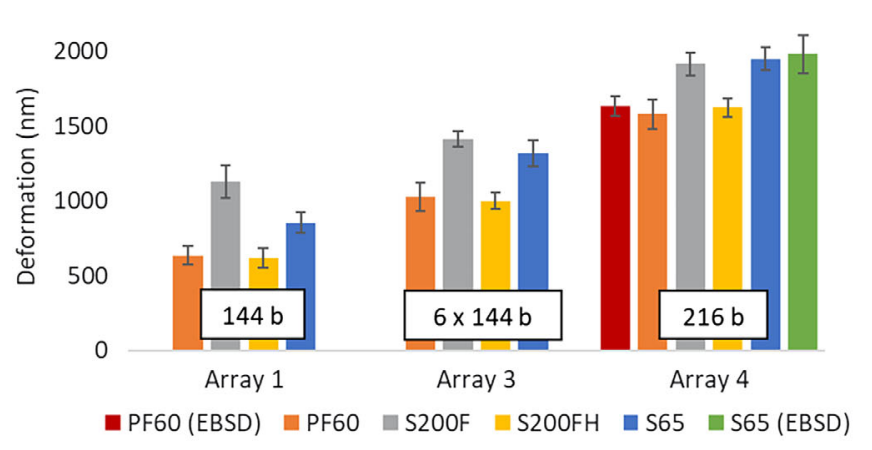

(a)

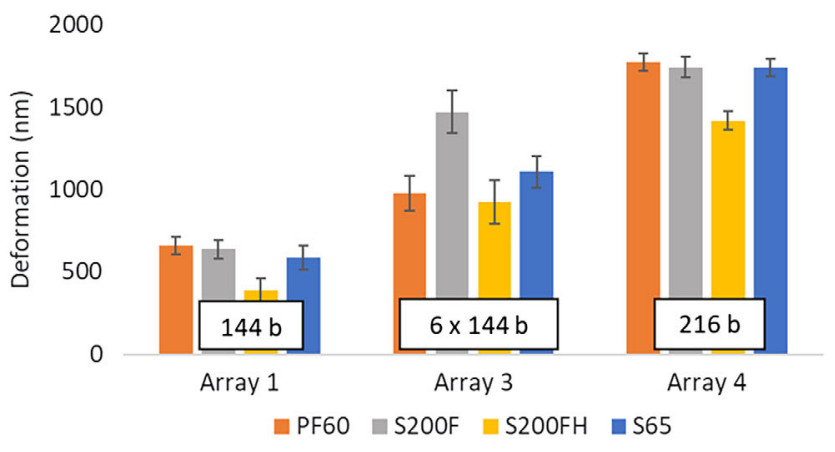

(b)

FIG. 10. Peak out-of-plane profilometry measurements of (a) 0.75-mm-thick and (b) 2-mm-thick beryllium disks.

strength reported for S-200-FH when compared to the other grades (Table I), thus incurring the least amount of plastic deformation. On the other hand, the smallest yield strength of the S-200-F grade leads to higher plastic deformation. Disks in array 3, which were exposed to multiple beam pulses $(6 \times 144$-bunch pulses) at the same location, confirm the effect of plastic strain ratcheting, where plastic deformation accumulates upon cyclic loading. Results show higher plastic deformations than for specimens in array 1, where only a single 144-bunch pulse was imposed. The following sections further analyze the out-of-plane deformation profile measurements and the benchmarking of numerical simulations based on a newly developed nonlinear strength model for the S-200-FH grade beryllium.

\section{NUMERICAL VALIDATION OF EXPERIMENTAL RESULTS}

\section{A. Development of beryllium Johnson-Cook strength model}

A highly nonlinear beryllium strength model was developed and implemented to help benchmark finite element analysis results with the experimentally measured beaminduced permanent out-of-plane deformations on the surface of the beryllium disks. The Johnson-Cook model [19] was chosen for this application, as it accounts for both strain rate and temperature effects on the material flow stress, which are key variables to accurately model beaminduced material response (high strain rates and temperatures). The yield stress of the model which incorporates strain hardening, strain rate, and thermal softening effects are defined by

$$
\sigma_{Y}=\left[A+B\left(\varepsilon_{\mathrm{eff}}^{p}\right)^{n}\right] \cdot\left[1+C \ln \varepsilon^{*}\right] \cdot\left[1-T_{H}^{m}\right],
$$

where $\varepsilon_{\text {eff }}^{p}$ is the equivalent plastic strain, $\dot{\varepsilon}^{*}=\dot{\varepsilon}_{\text {eff }}^{p} / \dot{\varepsilon}_{o}$ is the dimensionless plastic strain rate (generally, $\dot{\varepsilon}_{o}=1 \mathrm{~s}^{-1}$ ), $A, B, C, n$, and $m$ are material constants determined experimentally, $T_{H}=\frac{T-T_{R}}{T_{M}-T_{R}}$ is the homologous temperature, $T_{M}$ is the melting temperature $(1558 \mathrm{~K}$ for $\mathrm{Be})$, and
$T_{R}$ is the reference temperature when determining $A, B$, and $n$ (293 K in our case)

\section{Evaluation of Johnson-Cook strength model parameters}

Split Hopkinson pressure bar experiments at elevated strain rates and temperatures were performed by Southwest Research Institute (SwRI) on grade S-200-FH grade beryllium to evaluate the material parameters of the JohnsonCook strength model. Tension and compression tests were carried out at $20^{\circ} \mathrm{C}, 300^{\circ} \mathrm{C}, 500^{\circ} \mathrm{C}$, and $600^{\circ} \mathrm{C}$ with strain rates of $10^{-5} \mathrm{~s}^{-1}$ and up to $10^{3} \mathrm{~s}^{-1}$. The tests revealed that only compression tests provided significant information on the plasticity of the S-200-FH material, and, based on SwRI's previous experience in characterizing the JohnsonCook model for various materials, the compression test results were mainly used to derive the model parameters. The resulting Johnson-Cook strength parameters are listed in Table III along with other relevant material properties for S-200-FH beryllium.

\section{Finite element model implementation}

A 3D finite element model was created based on the beryllium disk geometries used in the experiment (diameter

TABLE III. Johnson-Cook model parameters in LS-DYNA ${ }^{\circledR}$ for S-200-FH grade beryllium.

\begin{tabular}{lc}
\hline \hline Parameters & \\
\hline$\rho_{o}$ & $1821 \mathrm{~kg} / \mathrm{m}^{3}$ \\
$G$ & $138 \mathrm{GPa}$ \\
$K(\mathrm{c} 1$ in EOS $)$ & $115 \mathrm{GPa}$ \\
$A$ & $432 \mathrm{MPa}$ \\
$B$ & $1280 \mathrm{MPa}$ \\
$C$ & 0.009 \\
$N$ & 0.5 \\
$M$ & 1.3 \\
$P_{\text {cutoff }}$ & $-10^{12}$ \\
SPALL & 1 \\
\hline \hline
\end{tabular}


of $15 \mathrm{~mm}$ with thicknesses of 0.75 and $2 \mathrm{~mm}$ ). Mesh optimization was carried out using ANSYS ${ }^{\circledR}$ workbench [20] multizone method to create a finer mesh around the beam center and a relatively larger mesh away from it, while adequate layers of elements were created through the thickness of the disk to capture the expected stress gradients. A minimum of 15 elements were created within one sigma of the beam spot in the radial direction to ensure that a smooth radial temperature profile was generated.

A two-step analysis was carried out for the thermalstructural simulation using ANSYS ${ }^{\circledR}$ workbench and LSDYNA ${ }^{\circledR}$. As ANSYS ${ }^{\circledR}$ workbench does not support implicit analysis with the Johnson-Cook model, only the transient thermal analysis was carried out in ANSYS ${ }^{\circledR}$ workbench, after which the results were exported to LS-DYNA ${ }^{\circledR}$ for the structural analyses with the Johnson-Cook model. For the thermal analysis, volumetric energy deposition from the proton beam interaction with the material was first calculated by the radiation physics code MARS [10] based on the Monte Carlo method. The nodal time-dependent temperature results from ANSYS ${ }^{\circledR}$ were then exported to LSDYNA ${ }^{\circledR}$ where the MAT15 Johnson-Cook strength material model was implemented. The damage and spallation modeling features in this material card were turned off in the simulations, as no damage parameters were developed for the material. A high negative value for pressure cutoff, $1 \times 10^{12}$ and SPALL $=1$, was selected to avoid the spallation algorithm and to allow the full range of stress calculation in the tensile as well as compressive regimes. This model also required an equation of state (EOS) for the material in order to properly capture the hydrodynamic behavior. Since we do not expect the pressure generated due to thermal shock to change the material's density significantly, a simple EOS based on the material bulk modulus was chosen for our simulation. Simulations were carried out for beryllium disks with two different thicknesses $(0.75$ and $2 \mathrm{~mm})$ exposed to two different beam intensity pulses (144 and 216 bunches).

\section{Profilometry raw data processing}

The surface profile measurements from the Alicona InfiniteFocus [18] optical profilometer produced a point cloud of 2.5 million data points with a grid spacing of $2.5 \mu \mathrm{m}$. The initial raw data analysis showed a lot of noise including a baseline noise of $0.5-1.0 \mu \mathrm{m}$, short-range noise with a peak value of $0.5 \mu \mathrm{m}$ spaced at about $50 \mu \mathrm{m}$, and medium-range artifacts at regular intervals of $500 \mu \mathrm{m}$ which may have compromised the actual out-of-plane displacement profile measurement near the beam center. As a result, filtering out of the short- and medium-range noise was necessary before comparing the experimental displacement profile and magnitude with numerical results. A MATLAB ${ }^{\circledR}$ software routine was written to process the raw data and fit to a high-order polynomial fit. After several iterations, a polynomial fit function of the tenth order was

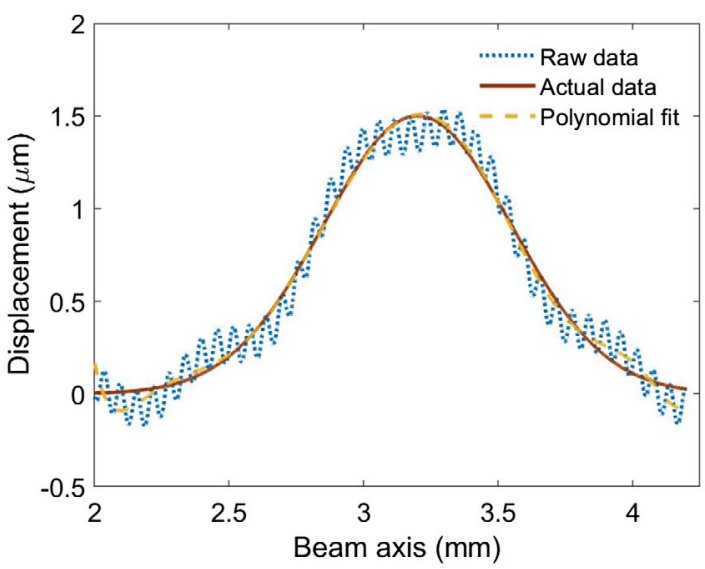

FIG. 11. Efficacy of a higher-order polynomial fit for raw profilometry data.

determined to be the most effective at fitting the raw data to remove the noise in the displacement profile, as shown in Fig. 11.

The final displacement profile was obtained by averaging multiple data paths (over $50 \mu \mathrm{m}$ ) along the major axis of the elliptical shape of the beam and used to compare with the corresponding displacement profile from the coupled ANSYS ${ }^{\circledR}$ and LS-DYNA ${ }^{\circledR}$ numerical simulations.

\section{B. Results}

Figure 12 shows a comparison of the out-of-plane displacement profiles along the beam spot major axis between experimental measurements and simulation results. Since the Johnson-Cook model parameters were developed for the S-200-FH beryllium grade, a comparison with experimental results is accurate only for that grade. As can be seen in the plots in Fig. 12, the simulation results match generally well with the experimental data in terms of the peak and shape of the displacement profile.

However, for the 2-mm-thick specimens exposed to 216 bunches in array 4, the numerical simulation underpredicts by about $20 \%$. It should be noted that the SPS beam emittance prior to extraction to the HiRadMat experimental area could not be measured for the highest-intensity beam pulse (array 4, 216 bunches) due to the beam intensity operational limit set on the wire scanner device. The beam spot size shown in Table II for array 4 (pulse 11) is the average of the beam spot sizes measured for array 3 (pulses 5-10). As a result, discrepancies between FEA results and profilometry measures for specimens in array 4 may be attributed to the uncertain beam spot size for pulse 11 . Further FEA analyses showed a high sensitivity of the beam spot size on the displacement profile peak where a $10 \%$ reduction in beam sigma led to an almost $50 \%$ increase in the peak displacement magnitude. Therefore, a small variation in the beam sigma can influence the resulting displacement profile quite significantly. 


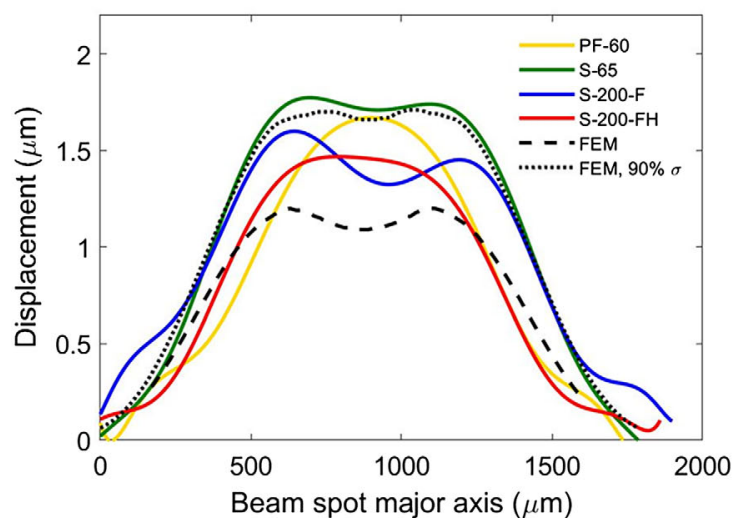

(a)

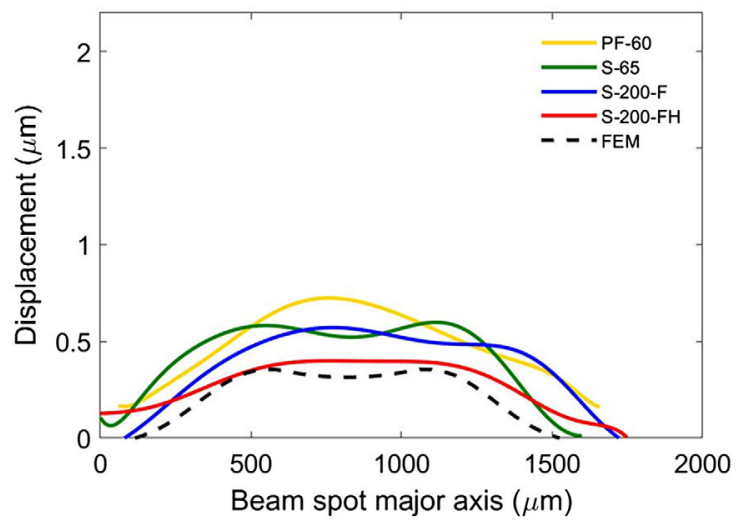

(c)

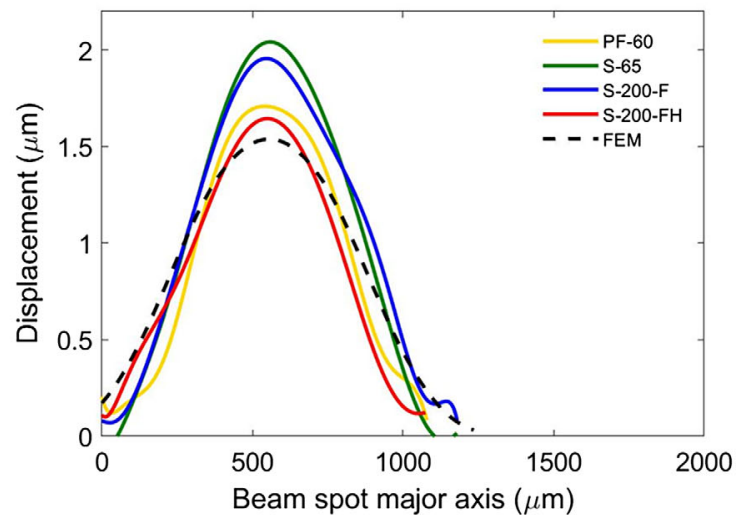

(b)

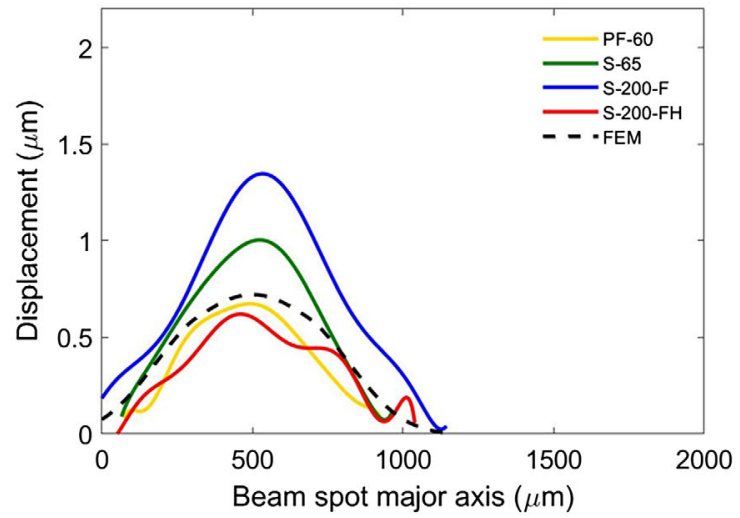

(d)

FIG. 12. Comparison of numerical results with displacement profile measurements along the beam spot major axis. (a) Array 4 (pulse 11, $2 \mathrm{~mm}$ disk), (b) array 4 (pulse 11, $0.75 \mathrm{~mm}$ disk), (c) array 1 (pulse 4, $2 \mathrm{~mm}$ disk), and (d) array 1 (pulse 4, $0.75 \mathrm{~mm}$ disk).

The relatively good agreement between numerical results and experimental measurements primarily indicates that the flow stress characteristics of the S-200-FH beryllium grade over the range of temperatures and stresses induced in the specimens have been accurately captured in the numerical simulations. Figure 13 shows how the yield stress of the S-200-FH beryllium grade material, calculated from the Johnson-Cook model, varies with the strain rate and temperature. The shaded red area on the plot indicates the range of strain rates and temperatures that were induced in the beryllium during this experiment as well as those expected in future accelerator beam-intercepting devices. As evident in Fig. 13, the yield stress can increase by up to $20 \%$ at high strain rates for different temperatures. Therefore, it is important to consider the strain rate effect in simulations and predictions of material mechanical response from beam-induced thermal shock.

The actual displacement profiles for the different beryllium grades show more variation in the peak magnitude than in profile widths. This variation may be attributed to differences in the material yield strengths of the different grades. In all cases, irrespective of beam intensities and the thickness of specimens, it is observed that grade S-200-FH has the minimum displacement of all grades, as it has the highest yield strength. In order to further investigate this observation, a sensitivity analysis of Johnson-Cook parameters was carried out to understand differences in the displacement response of the different grades. Figure 14

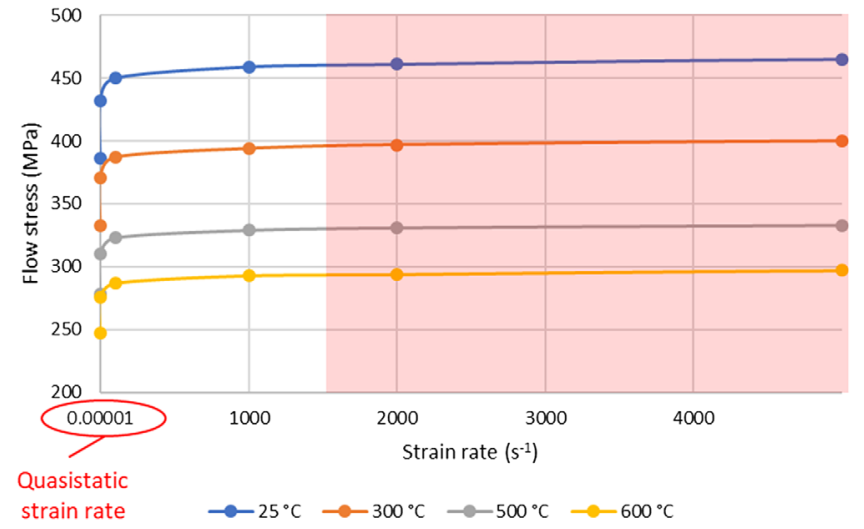

FIG. 13. Flow stress of beryllium grade S-200-FH as a function of the strain rate and temperature. 


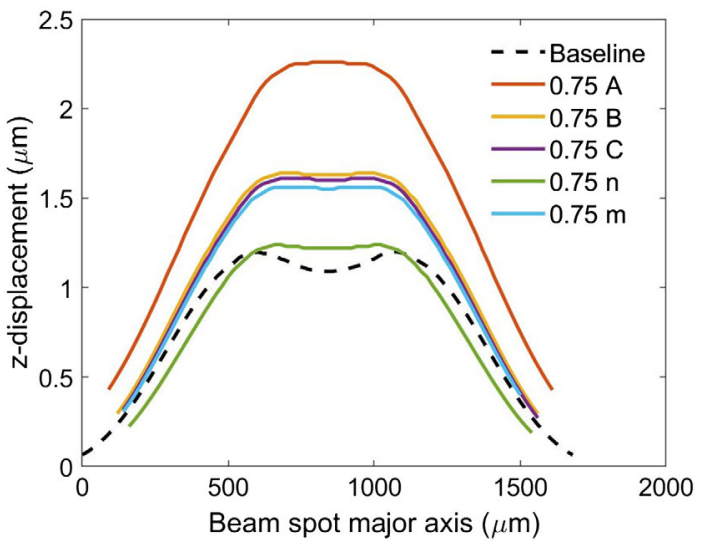

FIG. 14. Sensitivity analysis of Johnson-Cook parameters (array 4, pulse 11, $2 \mathrm{~mm}$ disk).

shows a sensitivity displacement profile response after changing each of the Johnson-Cook parameters by $25 \%$. Each of the material constants $B, C$, and $m$, which correspond to the hardening coefficient, strain rate coefficient, and temperature index respectively, has a significant effect on the peak displacement magnitude. However, the material yield strength parameter $A$ clearly has the largest influence on the peak displacement magnitude. This therefore indicates the importance of using strain-rateand temperature-dependent yield properties to improve the accuracy of simulation results of beam-intercepting devices.

Even though no surface cracking of the beryllium specimens were observed, there is a possibility that microcracking inside the specimens near the beam spot occurred.
If that is the case, the microcracks would also contribute to the amount of residual deformation measured by profilometry. This is another factor to consider when comparing the profilometry measurements with the Johnson-Cook (without damage model) numerical simulations.

Figure 12 also shows the dependence of the displacement profile shape on the specimen thickness. Thicker samples display a relatively flat top and broader bump size irrespective of beam intensities, evident in both profilometry measurements and numerical simulations. This may be explained by the effective plastic strain and stress triaxiality factor distributions within the specimen, as shown in Fig. 15. For thicker specimens, the volume under the beam center undergoes a negligible plastic deformation which is corroborated by higher stress triaxiality values in that region (higher ratio of mean stress to Von-Mises stress). A higher stress triaxiality indicates that the region is under considerable hydrostatic stress, leading to minimal distortion and hence less plastic deformation. The plastic strain distribution shows that the region a little away from the beam center has undergone a plastic deformation, while the central part has elastically recovered. Therefore, the displacement profile reveals a flat top between the shoulders of the plastically deformed region on either side of the beam center. High stress triaxiality also indicates that the region is in a three-dimensional state of stress (plane strain condition) which resists plastic deformation. In the case of the thinner specimens, stress triaxiality is negligible, corresponding to a situation of the plane stress condition where the plastic zone encompasses the total thickness of the sample. Thus, the maximum plastic strain is formed under the beam center in the thinner specimens, with a more rounded peak displacement profile (no flat top).

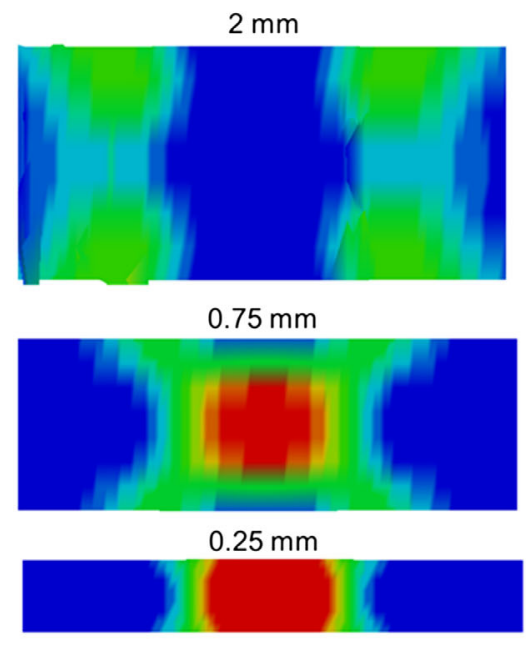

(a)

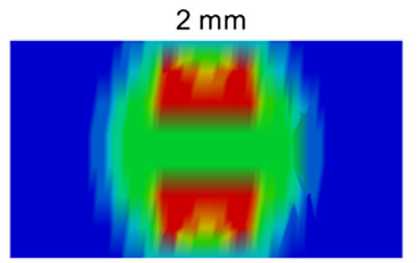

$0.75 \mathrm{~mm}$

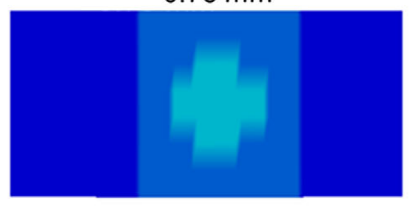

$0.25 \mathrm{~mm}$

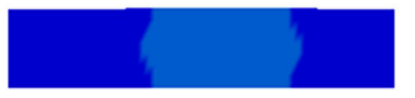

(b)

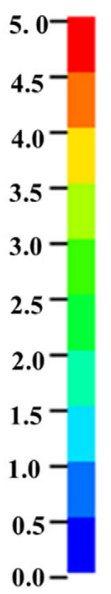

$0.0-$

FIG. 15. (a) Effective plastic strain and (b) triaxiality factor distribution, on a cross-sectional plane passing through the beam spot major axis at the end of cooldown after a 216-bunch pulse. 


\section{CONCLUSIONS}

The thermal shock response of various commercially available beryllium grades, induced by high energy highintensity proton beams at CERN's HiRadMat facility, was successfully and safely investigated. The experiment was designed to test and push beryllium to its failure limit, and, even though lower than desired beam intensities were attained, the degree of thermal shock induced was still larger than beryllium material is currently exposed to in currently operational target facilities. The online measurements and PIE results from this experiment provided valuable information and insight on the complex beaminduced thermomechanical response of the different beryllium grades.

For the nearly instantaneous maximum temperature jump of about $640^{\circ} \mathrm{C}$ imposed in this experiment, no surface cracks or failure in the beryllium disks were observed via optical microscopy. This, however, does not rule out the possibility of microcracks on the interior of the specimens which were not visible with optical microscopy. Profilometry measurements revealed a varying degree of induced plastic strain deformation, as exhibited by out-of-plane surface deformations between the different beryllium grades. The S-200-FH grade, due to its higher reported yield strength, was shown to consistently exhibit the least amount of plastic deformation, compared to the other beryllium grades. Furthermore, plastic strain ratcheting due to cyclic loading from the beam was confirmed and measured by the magnitude of the out-of-plane deformations in the specimens from array 3. Differences between the out-of-plane deformation profile with respect to the thickness of the specimen were observed from the profilometry measurements and attributed to be a function of the stress triaxiality distribution around the beam spot region.

The experiment's objective of benchmarking numerical models with measurements was also successfully achieved. A newly developed Johnson-Cook model for S-200-FH beryllium was validated with experimental measurements. The numerical results showed relatively good agreement with profilometry surface profile measurements, which now provides better confidence in simulating the thermal shock response of current and future S-200-FH beryllium components. The benchmarking results, using the JohnsonCook model, also indicated the importance of accurately considering the strain rate and temperature dependency in determining the yield stress of the material. As shown for beryllium S-200-FH grade in Fig. 13, the yield stress at elevated strain rates can be up to about $20 \%$ higher than the quasistatic yield point. As a result, a higher yield point can provide an extra margin in the design of future higherintensity beam-intercepting devices and is an important aspect to consider to avoid compromising secondary particle production efficiency by limiting beam parameters on such devices. Another essential factor to consider when determining safety margins for beam-intercepting devices is the long-term radiation damage effects on material properties. Previous studies [13,21,22] have shown a significant degradation in thermal and strength properties of beryllium from high energy particle irradiation, which can have a negative impact on the structural and thermal integrity of the component over time. Therefore, careful consideration of radiation damage effects and the resulting material property degradation is needed when evaluating the thermomechanical response of beam-intercepting devices.

The challenges faced during the execution of this experiment included lower than desired beam pulse intensities (larger beam size and lower bunch intensity), as well as a possible misalignment of the slug specimens during real-time measurements of the strain and temperature. Therefore, a follow-up experiment (HRMT-43) at the HiRadMat facility has been executed during 2018 to address these issues and to also incorporate the unique aspect of comparing the thermal shock response of previously proton-irradiated materials (irradiation-induced damaged materials) [23] with nonirradiated materials. Finally, to improve benchmarking of numerical simulations with experimental measurements, the development of the Johnson-Cook damage model for beryllium is desired. This will provide the ability to predict failure and crack initiation (microcracking inside of the material) of the material and more accurately simulate the expected out-of-plane deformation and structural response of the material.

\section{ACKNOWLEDGMENTS}

This manuscript has been authored by Fermi Research Alliance, LLC under Contract No. DE-AC02-07CH11359 with the U.S. Department of Energy, Office of Science, Office of High Energy Physics. In addition, the European Commission under the FP-7 Research Infrastructures project EuCARD-2, Grant Agreement No. 312453, provided support for this experiment.

[1] P. Hurh, K. Ammigan, B. Hartsell, and R. Tschirhart, Targetry challenges at megawatt proton accelerator facilities, in Proceedings of the 4th International Particle Accelerator Conference, IPAC-2013, Shanghai, China, 2013 (JACoW, Shanghai, China, 2013), THPFI082, pp. 3484-3486.

[2] T. R. Davenne and P. Loveridge, Propagation of elastic pressure waves in a beam window, Phys. Rev. Accel. Beams 19, 093501 (2016).

[3] Long Baseline Neutrino Facility (LBNF), http://lbnf.fnal .gov.

[4] C. J. Densham et al., Conceptual design study of the Long Baseline Neutrino Experiment (LBNE) target and beam window, final report, 2010.

[5] ITER fusion test reactor, https://www.iter.org/.

[6] J. Linke, R. Duwe, A. Gervash, R. H. Qian, M. Rodig, and A. Schuster, Material damage to beryllium, carbon and 
tungsten under severe thermal shocks, J. Nucl. Mater. 258-263, 634 (1998).

[7] B. Spilker, J. Linke, G. Pintsuk, and M. Wirtz, Impact of the surface quality on the thermal shock performance of beryllium armor tiles for first wall applications, Fusion Eng. Des. 109-111, 1692 (2016).

[8] K. Ammigan and P. Hurh, Status and update of the RaDIATE collaboration R\&D program, in Proceedings of the 13th International Topical Meeting on Nuclear Applications of Accelerators (AccApp17), Quebec, Canada, 2017, pp. 326-333, http://accapp17.org/wp-content/2017/ data/pdfs/144-22798.pdf.

[9] I. Efthymiopoulos, C. Hessler, H. Gaillard, D. Grenier, M. Meddahi, P. Trilhe, A. Pardons, C. Theis, N. Charitonidis, S. Evrard, H. Vincke, and M. Lazzaroni, HiRadMat: A new irradiation facility for material testing at CERN, in Proceedings of the 2nd International Particle Accelerator Conference, San Sebastián, Spain (EPS-AG, Spain, 2011), TUPS058.

[10] N. V. Mokhov and C. C. James, The MARS code system user's guide, version 15 (2016), Report No. Fermilab-FN1058-APC, 2017; N. V. Mokhov et al., MARs15 code developments driven by the intensity frontier needs, Prog. Nucl. Sci. Technol., 4, 496 (2014); https://mars.fnal.gov.

[11] D. Montoya et al., Comportement dynamique d'une nuance de beryllium, J. Phys. IV (France) 1, 27 (1991).

[12] Livermore Software Technology Corporation, LS-DYNA Keyword User's Manual Vol. 1, Rev. 5471, 2014.

[13] R. Chaouadi et al., Tensile and fracture toughness test results of neutron irradiated beryllium, ITER Task T23 report, 1997.

[14] S. Burger et al., Scintillation and OTR screen characterization with a $440 \mathrm{GeV} / \mathrm{c}$ proton beam in air at the CERN HiRadMat facility, in Proceedings of the International
Beam Instrumentation Conference (IBIC), Barcelona, Spain, MPOG78, pp. 268-272.

[15] T. Bogey and R. Jones, The beam position system of the CERN Neutrino to Grand Sasso proton beam line, in Proceedings of the 8th European Workshop on Beam Diagnostics and Instrumentation for Particle Accelerators (DIPAC 2007), Venice, Italy, TUPB31, pp. 141-143.

[16] Materion, Designing and fabricating beryllium, https://materion.com/-/media/files/pdfs/beryllium/berylliummaterials/mb-001designingandfabricatingberyllium.pdf.

[17] Materion brush beryllium and composites material certificates, S-65 lot No. 5326, S-200-FH lot No. H2148, and S-200-F lot No. 5321, 2013.

[18] Alicona InfiniteFocus, https://www.alicona.com/products/ infinitefocus/.

[19] G. R. Johnson and W. H. Cook, A constitutive model and data for metals subjected to large strains, high strain rates and high temperatures, in Proceedings of the Seventh International Symposium of Ballistics (The Hague, Netherlands, 1983).

[20] ANSYS $^{\circledR}$ Workbench, release 16.1, ANSYS, Inc.

[21] V. Chakin, J. Reimann, A. Moeslang, R. Latypov, and A. Obukhov, Thermal conductivity of highly neutronirradiated beryllium in nuclear fusion reactors, Prog. Nucl. Energy 57, 2 (2012).

[22] V. Kuksenko, K. Ammigan, B. Hartsell, C. Densham, P. Hurh, and S. Roberts, Irradiation effects in beryllium exposed to high energy protons of the NuMI neutrino source, J. Nucl. Mater. 490, 260 (2017).

[23] K. Ammigan et al., The RaDIATE high-energy proton materials irradiation experiment at the Brookhaven Linac isotope producer facility, in Proceedings of the Eight International Particle Accelerator Conference (IPAC17), Copenhagen, Denmark, 2017, WEPVA138, pp. 3593-3596. 